\title{
A Mass Spectrometric Analysis Method Based on PPCA and SVM for Early Detection of Ovarian Cancer
}

\author{
Jiang Wu, ${ }^{1}$ Yanju Ji, ${ }^{1}$ Ling Zhao, ${ }^{2}$ Mengying Ji, ${ }^{1}$ Zhuang Ye, $^{2}$ and Suyi Li ${ }^{1}$ \\ ${ }^{1}$ College of Electrical Engineering and Instrumentation, Jilin University, Changchun 130061, China \\ ${ }^{2}$ First Hospital, Jilin University, Changchun 130021, China \\ Correspondence should be addressed to Yanju Ji; jiyj@jlu.edu.cn and Suyi Li; lisusu9911@qq.com
}

Received 21 April 2016; Revised 17 June 2016; Accepted 13 July 2016

Academic Editor: Erik Cuevas

Copyright (c) 2016 Jiang Wu et al. This is an open access article distributed under the Creative Commons Attribution License, which permits unrestricted use, distribution, and reproduction in any medium, provided the original work is properly cited.

\begin{abstract}
Background. Surfaced-enhanced laser desorption-ionization-time of flight mass spectrometry (SELDI-TOF-MS) technology plays an important role in the early diagnosis of ovarian cancer. However, the raw MS data is highly dimensional and redundant. Therefore, it is necessary to study rapid and accurate detection methods from the massive MS data. Methods. The clinical data set used in the experiments for early cancer detection consisted of 216 SELDI-TOF-MS samples. An MS analysis method based on probabilistic principal components analysis (PPCA) and support vector machine (SVM) was proposed and applied to the ovarian cancer early classification in the data set. Additionally, by the same data set, we also established a traditional PCA-SVM model. Finally we compared the two models in detection accuracy, specificity, and sensitivity. Results. Using independent training and testing experiments 10 times to evaluate the ovarian cancer detection models, the average prediction accuracy, sensitivity, and specificity of the PCA-SVM model were $83.34 \%, 82.70 \%$, and $83.88 \%$, respectively. In contrast, those of the PPCA-SVM model were $90.80 \%, 92.98 \%$, and $88.97 \%$, respectively. Conclusions. The PPCA-SVM model had better detection performance. And the model combined with the SELDI-TOF-MS technology had a prospect in early clinical detection and diagnosis of ovarian cancer.
\end{abstract}

\section{Introduction}

The mortality of ovarian cancer ranks first in female genital malignancies; owing to the fact of being uneasy to find, the 5 -year survival rate is only about $30 \%$ [1]. Studies show that if ovarian cancer patients can get early diagnosis, the survival rate can be raised to about $90 \%$ [2]. Thus, early diagnosis and treatment are critical for improving the patients' cure rate and prolonging their survival.

Surfaced-enhanced laser desorption-ionization-time of flight mass spectrometry (SELDI-TOF-MS) is a new technology in proteomics research. For the accurately and quickly screening of large numbers of proteins within cells and tissues to identify specific tumor markers, it has a specific advantage in the early diagnosis of tumors [3-5].

However, the raw MS data is highly dimensional and redundant. Therefore, it is an important task to extract the features and establish a classification model in the massive MS data analysis. Currently MS data analysis methods mainly include pattern matching algorithm [6], genetic algorithm
[7], chi-square test [8], extended Markov blanket [9], principal component analysis [10], artificial neural network [11], partial least squares analysis [12], robust SVM [13], and some combination methods $[14,15]$, such as wavelet and ANN, PCA, and SVM, in which the combination of PCA and SVM method obtains best results. But the principal component analysis (PCA) is based on the minimum variance principle of reconstruction, leading to a lack of probabilistic model structure and high order statistics. Probability PCA (PPCA) restricts the factor loading matrix with a noise variance estimation using the principle components ignored by the traditional PCA and then obtains the optimal probability model through the estimated parameters by the expectation-maximization algorithm. Consequently, PPCA can find the direction of the principal components from the high-dimensional data more effectively and can obtain the outstanding features extraction efficiently [16]. Simultaneously, the performance of SVM generally outperforms that of other classifiers applied in nonlinear classification, including 


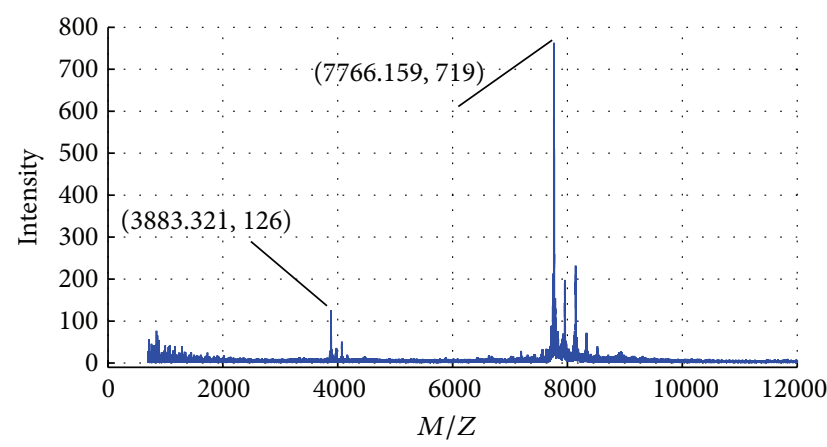

(a)

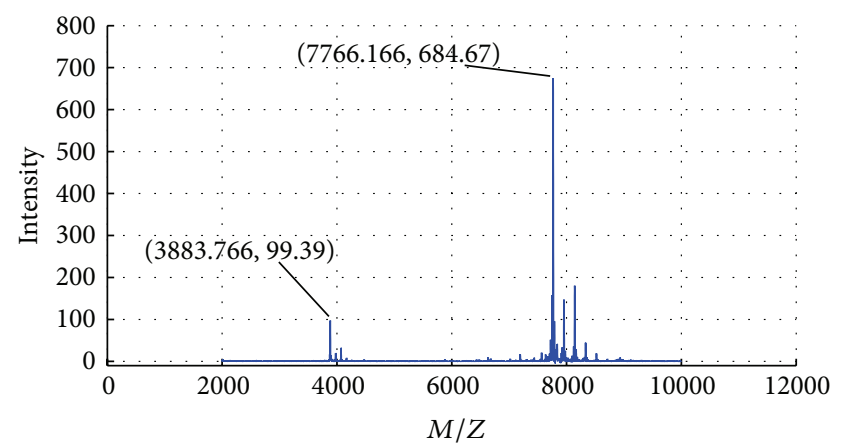

(c)

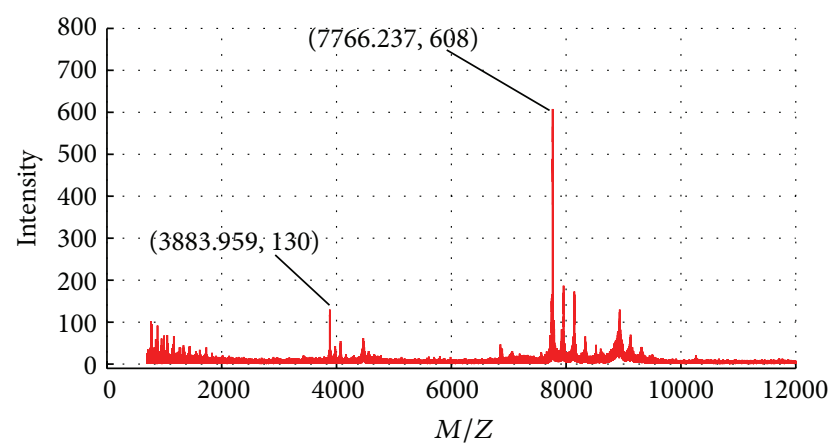

(b)

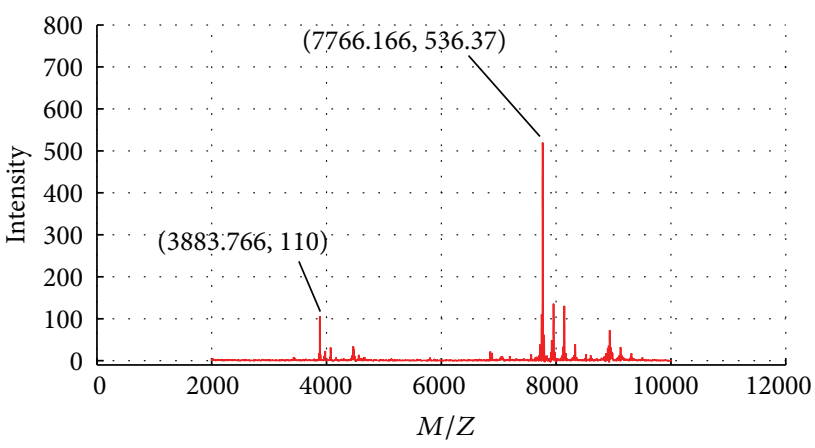

(d)

Figure 1: Comparison of SELDI-TOF-MS of serum from an unaffected individual (a) and from an ovarian cancer patient (b) and the corresponding preprocessing result of the unaffected individual (c) and that of the ovarian cancer patient (d).

iterative thresholding algorithm, self-organizing map, and $k$ nearest neighbor algorithm [17].

According to the above analysis, we focused on the design of an automatic model using PPCA and SVM technique for the ovarian cancer identification from MS data. In order to examine the performance of our proposed method, we established a PPCA-SVM model to classify ovarian cancer automatically and compared its average prediction accuracy, sensitivity, and specificity with those of a traditional PCASVM model using the same clinical data set.

\section{Material and Methods}

2.1. Data Set. The clinical data set used in this study was provided by the FDA-NCI center. By using the serum samples obtained by National Ovarian Cancer Early Detection Program (NOCEDP) and gynecologic oncology clinic at Northwestern University (Chicago, IL, USA), the FDA-NCI center formed the clinical data set via ProteinChip weak cation exchange interaction chips (WCX2, Ciphergen Biosystems, Inc., Fremont, CA, USA) and SELDI-TOF-MS technology [18]. The clinical data set consisted of 216 SELDI-TOF-MS samples, including 121 samples from ovarian cancer patients and 95 samples from healthy people.

The dimension of the raw SELDI-TOF-MS sample in feature space was high (each sample has about 360,000 features). Figure 1(a) showed the spectrum of a healthy sample and Figure $1(b)$ showed that of an ovarian cancer patient. Differences could be seen in intensity of cancer sample and healthy sample. In Figure 1(a), the intensities were 126 and 719 at $M / Z 3883.321$ and 7766.159 , respectively. In Figure 1(b), the intensities were 130 and 608 at $M / Z 3883.959$ and 7766.237, respectively.

From Figures 1(a) and 1(b), it can be seen that the valid information was concentrated between $M / Z 2000$ and $M / Z$ 10000 , and the raw spectrum contained a lot of redundancy and noise. Meanwhile, its prominent peaks needed to be aligned. Therefore, we employed the generally used preprocessing procedure to treat the raw data, including resampling, alignment, denoising, and normalization. The detailed description of the preprocessing procedure can be found in [5]. Figure 1(c) was the preprocessed spectrum of Figure 1(a) and Figure 1(d) was that of Figure 1(b). It can be seen that, after preprocessing, the dimension was reduced to 15000 , the prominent peaks were aligned, the background was corrected, and the noise was suppressed.

2.2. Feature Extraction Using PPCA. After the preprocessing stage, the SELDI-TOF-MS data set was still highly dimensional. Extracting features by using dimension reduction techniques not only simplifies the structure of the prediction model but also improves the speed of training and testing. PCA is a commonly used dimension reduction technique based on the minimum variance principle of reconstruction. What is more, it uses the small amount of principle components to replace the massive data. However, PCA is lack of probabilistic model structure and highly order statistics. PPCA, proposed by Tipping and Bishop [16], restricts the 
factor loading matrix with a noise variance estimation using the principle components ignored by the traditional PCA in the latent variable model and then obtains the optimal probability model through the parameters estimated by the expectation-maximization (EM) algorithm. Consequently, PPCA can find the direction of the principal components from the high-dimensional data more effectively and can obtain the outstanding feature extraction more efficiently.

Suppose that the dimension of an observation data set $\left\{S_{n}, n=1,2, \ldots, N\right\}$ is $d$ and the number of samples is $N$. For one sample, through the latent variable model, the relationship between the observation data $S$ and the latent variable $X$ can be expressed as

$$
S=W X+\mu+\varepsilon,
$$

where $W$ is a $d \times q$ factor loading matrix, $X$ is a $q$-dimensional latent variable, $\mu=(1 / N) \sum_{n=1}^{N} S_{n}$, is a nonzero mean, $\varepsilon$ is error and assume $X \sim N(0, I)$ and $\varepsilon \sim N\left(0, \sigma^{2} I\right)$, and then we can obtain the probability distribution of $S$ under the condition of $X$ through (1) as follows:

$$
p\left(\frac{S}{X}\right)=\left(2 \pi \sigma^{2}\right)^{-d / 2} \exp \left\{-\frac{1}{2 \sigma^{2}}\|S-W X-\mu\|^{2}\right\} .
$$

If the prior probability model of $X$ conforms to Gaussian distribution

$$
p(X)=(2 \pi)^{q / 2} \exp \left\{\frac{1}{2} X^{T} X\right\}
$$

then the probability distribution of $S$ can be expressed as

$$
\begin{aligned}
& p(S) \\
& \quad=(2 \pi)^{-d / 2}|C|^{-1 / 2} \exp \left\{-\frac{1}{2}(S-\mu)^{T} C^{-1}(S-\mu)\right\},
\end{aligned}
$$

where $C=W W^{T}+\sigma^{2} I$ is a $d \times d$ matrix. By using Bayes rule, we can derive the posterior probability distribution of $X$ from S:

$$
\begin{aligned}
& p\left(\frac{X}{S}\right) \\
& =(2 \pi)^{-q / 2} \sigma^{2} M^{-1 / 2} \exp \left\{-\frac{1}{2}(S-\mu)^{T} M^{-1}(S-\mu)\right\},
\end{aligned}
$$

where $M=W^{T} W+\sigma^{2} I$ is a $q \times q$ matrix. Under this model, the Log-likelihood function of $S$ can be expressed as

$$
L=-\frac{N}{2}\left\{d \ln (2 \pi)+\ln |C|+\operatorname{tr}\left(C^{-1} U\right)\right\}
$$

where $U=(1 / N) \sum_{n=1}^{N}\left(S_{n}-\mu\right)\left(S_{n}-\mu\right)^{T}$ is the covariance matrix of the observations, and then we can obtain the maximum likelihood estimates through the EM algorithm:

$$
\begin{aligned}
& \widetilde{W}=S W\left(\sigma^{2} I+M^{-1} W^{T} S W\right)^{-1}, \\
& \widetilde{\sigma}^{2}=\frac{1}{d} \operatorname{tr}\left(S-S W M^{-1} \widetilde{W}^{T}\right),
\end{aligned}
$$

where $W$ is the old value of the parameter matrix and $\widetilde{W}$ is the revised estimates calculated from (7). We bring the parameters obtained from (7) and (8) into (1) to derive the latent variable $\widetilde{X}_{n}$ which is the dimensionality reduction form of the observations $S_{n}$ :

$$
\widetilde{X}_{n}=\widetilde{W}^{T}\left(S_{n}-\mu\right)
$$
$\widetilde{X}_{n}$ :

From (9), we can reconstruct the observation data $\widetilde{S}_{n}$ via

$$
\widetilde{S}_{n}=\widetilde{W}(\widetilde{W} W)^{-1} \widetilde{X}_{n}+\mu
$$

2.3. SVM Model. SVM is derived from statistical learning theory. Its learning goal transforms empirical risk minimization into structure risk minimization and improves the overfitting problem [19]. In this study, the data set was under the PPCA dimensionality reduction procedure. And then we employed SVM technology to build an automatic detection model for ovarian cancer classification.

The implementation of the model establishment can be converted into solving the optimization as follows:

$$
\begin{array}{ll}
\text { Minimize } & \frac{1}{2} w^{t} w+c \sum_{i=1}^{N} \xi_{i} \\
\text { Subject to } & y_{n}\left[w^{t} \phi\left(x_{n}\right)+w_{0}\right] \geq 1-\xi_{n} \\
& n=1,2, \ldots, N,
\end{array}
$$

where $x_{n}$ is the dimensionality reduction data set after PPCA, $n$ is the number of samples, $c$ is a regularization constant, which determines the weigh between the maximum margin and the minimum classification error, $\xi_{n}$ is the slack variable, $y_{n}$ is the desired output, and $\phi\left(x_{n}\right)$ is the kernel function that maps nonlinear data into linear in high-dimensional space.

2.4. Implementation of the PPCA-SVM Classifier. In this study we used MATLAB R2013 software and Lib-SVM toolbox [20] to build the classifier, and the implementation steps are as follows.

Step 1 (selection of the training set and the prediction set). The preprocessed clinical data set included 216 samples; each sample had 15000 protein absorption features and had an appropriate type of clinical categories, negative for normal and positive for ovarian cancer patients.

We chose $70 \%$ of the data set randomly as the training set, the remaining as the prediction set.

Step 2 (feature extraction). We used PCA to reduce the dimension. The cumulative contribution rate could reach 99.99\% when using 215 principal vectors in PCA. So we applied PCA for feature extraction, reducing the data dimension from 15000 to 215 and PPCA for that using the same principal vectors.

Step 3 (SVM modeling). We employed SVM to establish the detection model and trained the SVM model using a radial 


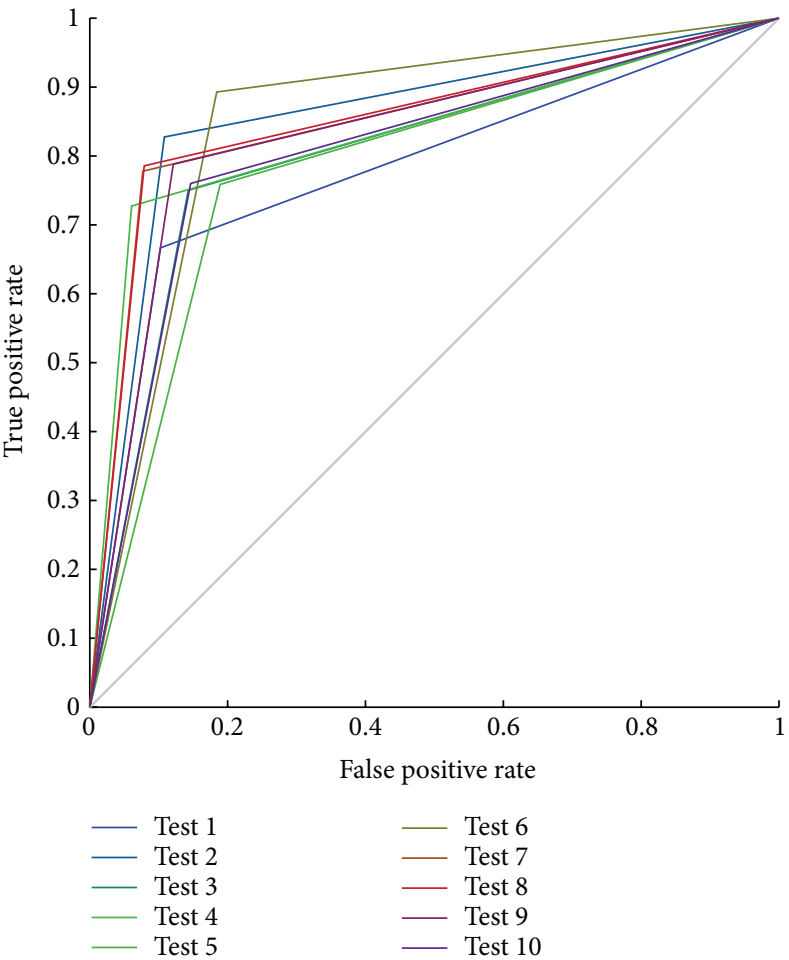

(a)

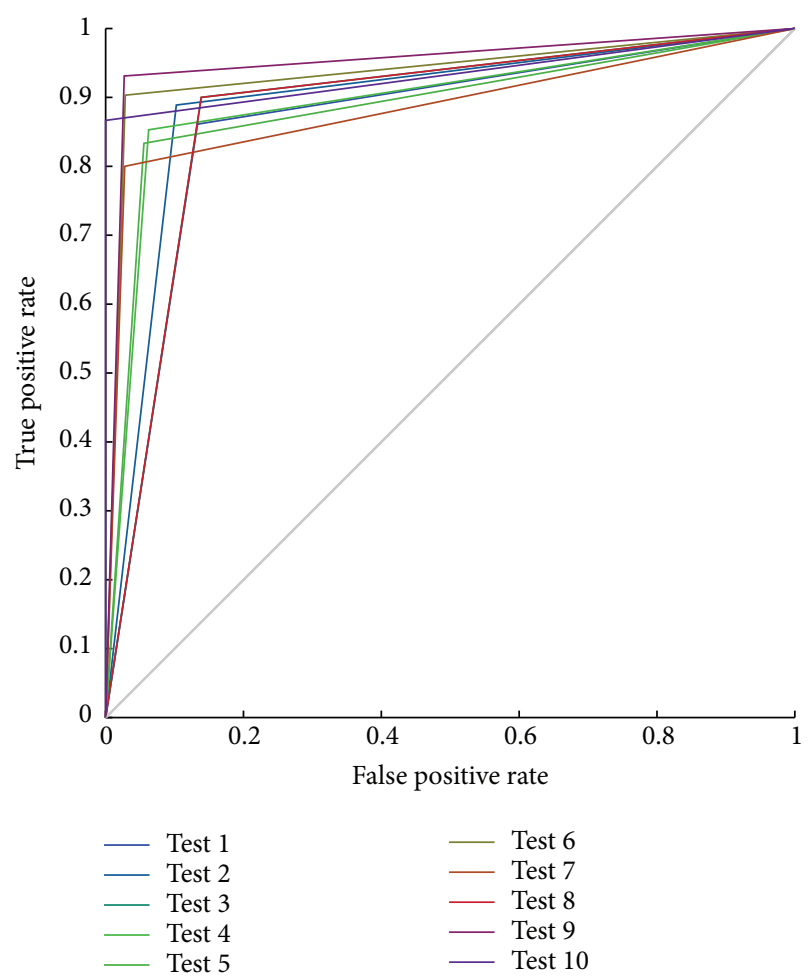

(b)

FIgURE 2: ROC graphic of PCA-SVM method (a) and ROC graphic of PPCA-SVM method (b).

basis function (RBF) kernel, which maps nonlinear data into a higher dimensional space. In order to obtain the optimal combination of penalty parameters, $c$ and $g$ of the RBF kernel, we conducted 10 -fold cross-validation based on the training set and then established SVM model by applying training set as input matrix and clinical categories as output matrix.

Step 4 (model evaluation). The detection model was established by using the training set. We used the prediction set to verify its performance. The evaluation parameters included the prediction accuracy $($ Accuracy $=((\mathrm{TP}+\mathrm{TN}) /(\mathrm{TP}+\mathrm{TN}+$ $\mathrm{FP}+\mathrm{FN})) \times 100 \%)$, the sensitivity (Sensitivity $=(\mathrm{TP} /(\mathrm{FN}+$ $\mathrm{TP})) \times 100 \%)$, and the specificity (Specifity $=(\mathrm{TN} /(\mathrm{FP}+$ $\mathrm{TN})$ ) $\times 100 \%$ ), where TP, TN, FP, and FN were the number of true positive, true negative, false positive, and false negative, respectively. To avoid accidental error, this experiment was repeated for 10 times.

\section{Results and Discussion}

Using the prediction set, we conducted the prediction experiments for 10 times and compared the evaluation parameters of the PPCA-SVM model and the PCA-SVM model, respectively. Table 1 showed the accuracy, sensitivity and specificity in classification.

Table 1 showed that the average prediction accuracy, the sensitivity, and the specificity of the PCA-SVM model were $83.34 \%, 82.70 \%$, and $83.88 \%$, respectively. In contrast, those of the PPCA-SVM model were $90.80 \%$, 92.98\%, and $88.97 \%$, respectively. The PPCA-SVM model obtained higher accuracy, sensitivity, and specificity, outperforming the PCASVM model.

To evaluate the accuracy of the classifier with binary outcomes, we also drew the receiver operating characteristic (ROC) curve of the PCA-SVM and the PPCA-SVM model, respectively. Figure 2(a) showed the ROC curves obtained under 10 prediction experiments using the PCA-SVM classifier, and Figure 2(b) showed that using the PPCA-SVM classifier.

It is known that, in ROC space, the closer to the upper left corner, the higher the forecast accuracy. Oppositely, the closer to the bottom right corner, the lower the accuracy. Comparing the ROC curves of the PCA-SVM (Figure 2(a)) with that of the PPCA-SVM classifier (Figure 2(b)), the distance between the upper left corner and the ROC curves in Figure 2(a) was less than that in Figure 2(b), which meant the PPCA-SVM classifier was superior to the PCA-SVM classifier.

\section{Conclusions}

Early diagnosis of ovarian cancer can significantly improve the patients' cure rate and prolong their survival time. SELDITOF-MS has been shown to be an efficient technique in the early diagnosis of tumors, which enjoys large numbers of proteins screening within cells and tissues to identify specific tumor markers accurately. In this study, we used 216 SELDITOF-MS samples of ovarian cancer patients and healthy people to research an automatic detection method which enjoyed 
TABLE 1: Comparison of the accuracy, sensitivity, and specificity of the PCA-SVM and of the PPCA-SVM model.

\begin{tabular}{|c|c|c|c|c|c|c|}
\hline & \multicolumn{3}{|c|}{ PCA-SVM prediction (\%) } & \multicolumn{3}{|c|}{ PPCA-SVM prediction (\%) } \\
\hline & Accuracy & Sensitivity & Specificity & Accuracy & Sensitivity & Specificity \\
\hline 1 & 80.30 & 81.81 & 79.54 & 87.87 & 87.50 & 88.09 \\
\hline 2 & 86.36 & 85.71 & 86.84 & 89.39 & 88.88 & 89.74 \\
\hline 3 & 81.81 & 75.00 & 85.71 & 86.36 & 93.33 & 80.55 \\
\hline 4 & 83.33 & 92.30 & 77.50 & 90.90 & 90.62 & 91.17 \\
\hline 5 & 78.78 & 75.86 & 81.08 & 89.39 & 92.59 & 87.18 \\
\hline 6 & 84.84 & 78.12 & 91.17 & 93.93 & 96.55 & 91.89 \\
\hline 7 & 86.36 & 87.50 & 85.71 & 89.39 & 96.00 & 85.36 \\
\hline 8 & 86.36 & 88.00 & 85.36 & 92.42 & 88.88 & 94.87 \\
\hline 9 & 83.33 & 86.66 & 80.55 & 92.42 & 95.45 & 90.90 \\
\hline 10 & 81.81 & 76.00 & 85.36 & 93.93 & 100 & 90.00 \\
\hline Average & 83.34 & 82.70 & 83.88 & 90.80 & 92.98 & 88.97 \\
\hline
\end{tabular}

higher prediction accuracy and efficiency and propose a PPCA-SVM classifier. To verify the model, we compared the accuracy, sensitivity, specificity, and ROC of the PPCA-SVM and those of the traditional PCA-SVM classifier through numerous experiments. The results indicated that the PPCASVM model was an accurate and effective model to identify the ovarian cancer, and the PPCA-SVM method combined with the SELDI-TOF-MS technology had a prospect in early clinical diagnosis of cancer.

\section{Abbreviations}

SELDI-TOF-MS: Surfaced-enhanced laser desorption-ionization-time of flight mass spectrometry

PCA: $\quad$ Principal components analysis

PPCA: $\quad$ Probabilistic principal components analysis

SVM: $\quad$ Support vector machine

NOCEDP: National ovarian cancer early

EM: $\quad$ Expectation-maximization

RBF: Radial basis function

TP: True positive

TN: $\quad$ True negative

FP: $\quad$ False positive

FN: $\quad$ False negative

ROC: $\quad$ Receiver operating characteristic.

\section{Competing Interests}

The authors declare that they have no competing interests.

\section{Authors' Contributions}

Yanju Ji, Jiang Wu, and Suyi Li conceived of this study and drafted the paper; Mengying Ji supplied the PPCA and PCA analysis results and also drafted the paper; Suyi Li designed the SVM model; Suyi Li verified the experimental results; Zhuang Ye and Ling Zhao gave some medical advice and MS data pretreatment. All authors contributed to the discussion of the work and approved the final paper.

\section{Acknowledgments}

This study was carried out within the framework of the project "An Automatic Detection Model of ECG-SAS Based on PCA-ANN (41174095)" supported by the National Natural Science Foundation of Jilin province, China. The authors thank the members of the projects committee for their help.

\section{References}

[1] American Cancer Society, Cancer Facts and Figures 2015, American Cancer Society, Atlanta, Ga, USA, 2015.

[2] U. Menon and I. Jacobs, "Ovarian cancer screening in the general population," Ultrasound in Obstetrics \& Gynecology, vol. 26, p. 243, 2012.

[3] E. F. Petricoin, A. M. Ardekani, B. A. Hitt, and P. J. Levine, "Use of proteomic patterns in serum to identify ovarian cancer," Physics, vol. 359, pp. 572-577, 2002.

[4] L. H. Cazares, B.-L. Adam, M. D. Ward et al., "Normal, benign, preneoplastic, and malignant prostate cells have distinct protein expression profiles resolved by Surface Enhanced Laser Desorption/Ionization mass spectrometry," Clinical Cancer Research, vol. 8, no. 8, pp. 2541-2552, 2002.

[5] T. P. Conrads, V. A. Fusaro, S. Ross et al., "High-resolution serum proteomic features for ovarian cancer detection," Endocrine-Related Cancer, vol. 11, no. 2, pp. 163-178, 2004.

[6] B.-L. Adam, Y. Qu, J. W. Davis et al., "Serum protein fingerprinting coupled with a pattern-matching algorithm distinguishes prostate cancer from benign prostate hyperplasia and healthy men," Cancer Research, vol. 62, no. 13, pp. 3609-3614, 2002.

[7] L. Li, D. M. Umbach, P. Terry, and J. A. Taylor, "Application of the GA/KNN method to SELDI proteomics data," Bioinformatics, vol. 20, no. 10, pp. 1638-1640, 2004.

[8] E. F. Petricoin III, D. K. Ornstein, C. P. Paweletz et al., "Serum proteomic patterns for detection of prostate cancer," Journal of the National Cancer Institute, vol. 94, no. 20, pp. 1576-1578, 2002.

[9] J. H. Oh, Y. Lotan, P. Gurnani, K. P. Rosenblatt, and J. Gao, "Prostate cancer biomarker discovery using high performance 
mass spectral serum profiling," Computer Methods and Programs in Biomedicine, vol. 96, no. 1, pp. 33-41, 2009.

[10] M. Lamberto and M. Saitta, "Principal component analysis in fast atom bombardment-mass spectrometry of triacylglycerols in edible oils," Journal of the American Oil Chemists' Society, vol. 72, no. 8, pp. 867-871, 1995.

[11] P. Johansson and M. Ringnér, "Artificial neural network for charge prediction in metabolite identification by mass spectrometry," in Classification of Genomic and Proteomic Data Using Support Vector Machines Fundamentals of Data Mining in Genomics and Proteomics, pp. 187-202, 2007.

[12] H. Gu, Z. Pan, B. Xi, V. Asiago, B. Musselman, and D. Raftery, "Principal component directed partial least squares analysis for combining nuclear magnetic resonance and mass spectrometry data in metabolomics: application to the detection of breast cancer," Analytica Chimica Acta, vol. 686, no. 1-2, pp. 57-63, 2011.

[13] E. Marchiori, C. R. Jimenez, M. West-Nielsen, and N. H. H. Heegaard, "Robust SVM-based biomarker selection with noisy mass spectrometric proteomic data," in Applications of Evolutionary Computing, F. Rothlauf, J. Branke, S. Cagnoni et al., Eds., vol. 3907 of Lecture Notes in Computer Science, pp. 79-90, Springer, New York, NY, USA, 2006.

[14] P. G. Lokhov, O. N. Kharybin, and A. I. Archakov, "Diagnosis of lung cancer based on direct-infusion electrospray mass spectrometry of blood plasma metabolites," International Journal of Mass Spectrometry, vol. 309, pp. 200-205, 2012.

[15] E. Suarez, H. P. Nguyen, I. P. Ortiz et al., "Matrix-assisted laser desorption/ionization-mass spectrometry of cuticular lipid profiles can differentiate sex, age, and mating status of Anopheles gambiae mosquitoes," Analytica Chimica Acta, vol. 706, no. 1, pp. 157-163, 2011.

[16] M. E. Tipping and C. M. Bishop, "Probabilistic principal component analysis," Journal of the Royal Statistical Society: Series B (Statistical Methodology), vol. 61, no. 3, pp. 611-622, 1999.

[17] C. C. Cheng, T. Y. Hsieh, J. S. Taur, and Y. F. Chen, "An automatic segmentation and classification framework for anti-nuclear antibody images," BioMedical Engineering OnLine, vol. 12, supplement 1, article S5, 2013.

[18] Clinical Proteomics Data Bank, 2014, https://home.ccr.cancer .gov/ncifdaproteomics/ppatterns.asp.

[19] N. Cristianini and J. Shawe-Taylor, Support Vector Machines and Other Kernel-Based Learning Methods, Cambridge University Press, Cambridge, Uk, 2000.

[20] C. C. Chang and C. J. Lin, "LIBSVM: a library for support vector machines," 2001, Software, http://www.csie.ntu.edu .tw/ cjlin/libsvm. 


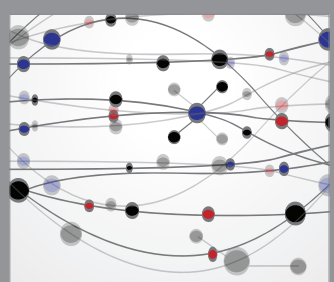

The Scientific World Journal
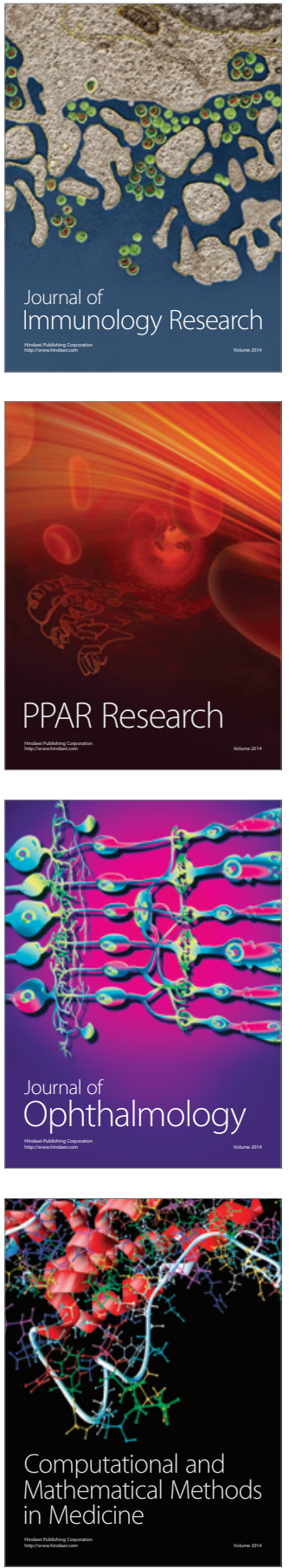

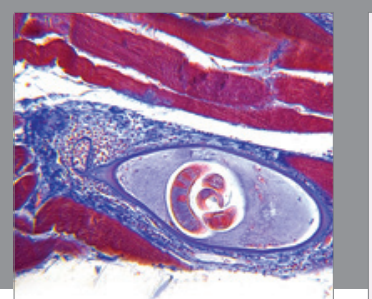

Gastroenterology Research and Practice

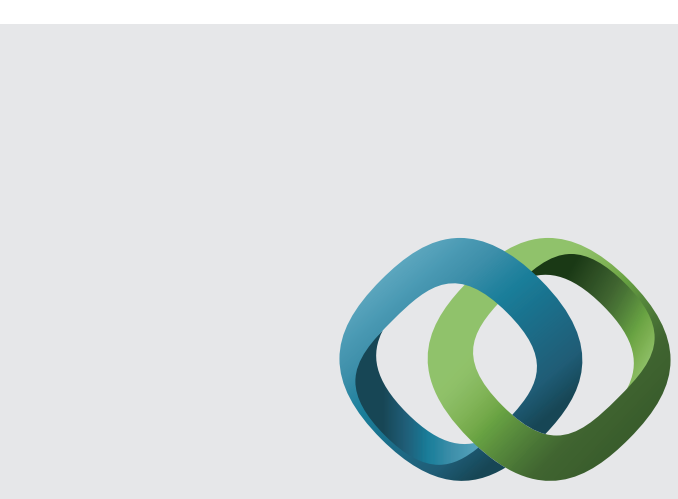

\section{Hindawi}

Submit your manuscripts at

http://www.hindawi.com
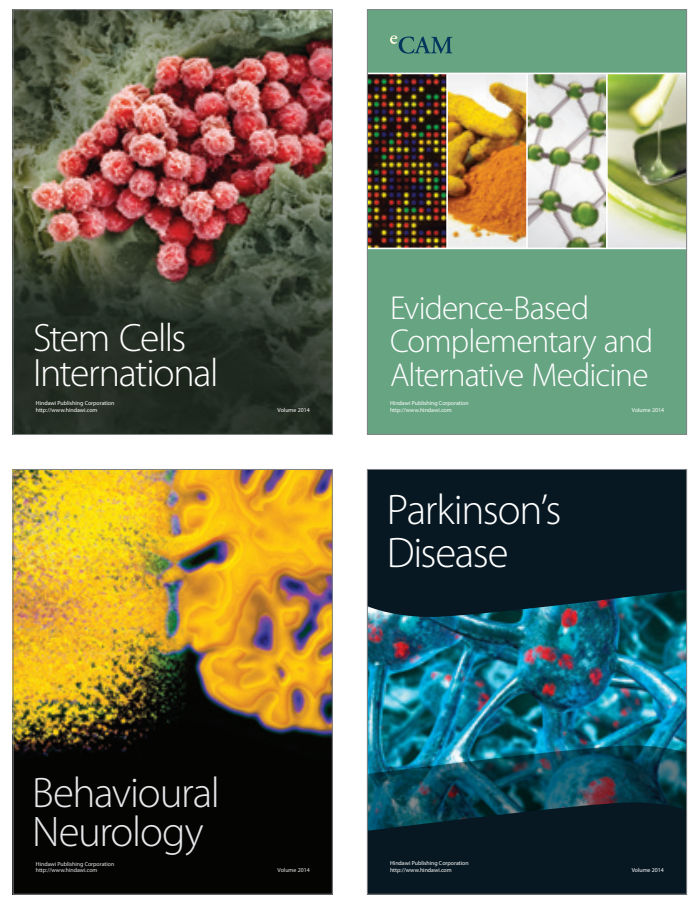
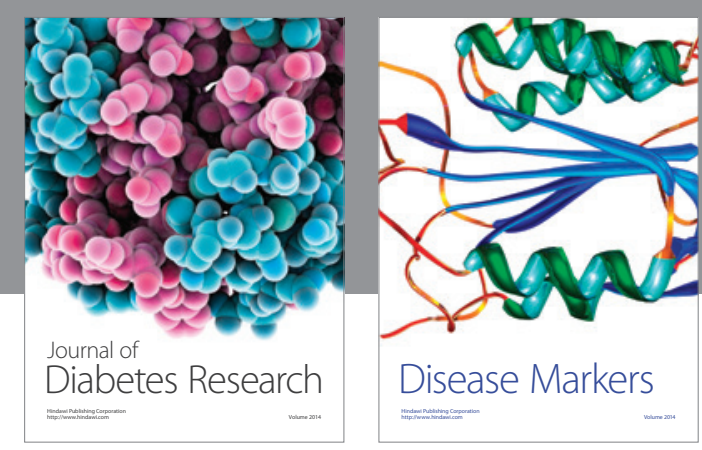

Disease Markers
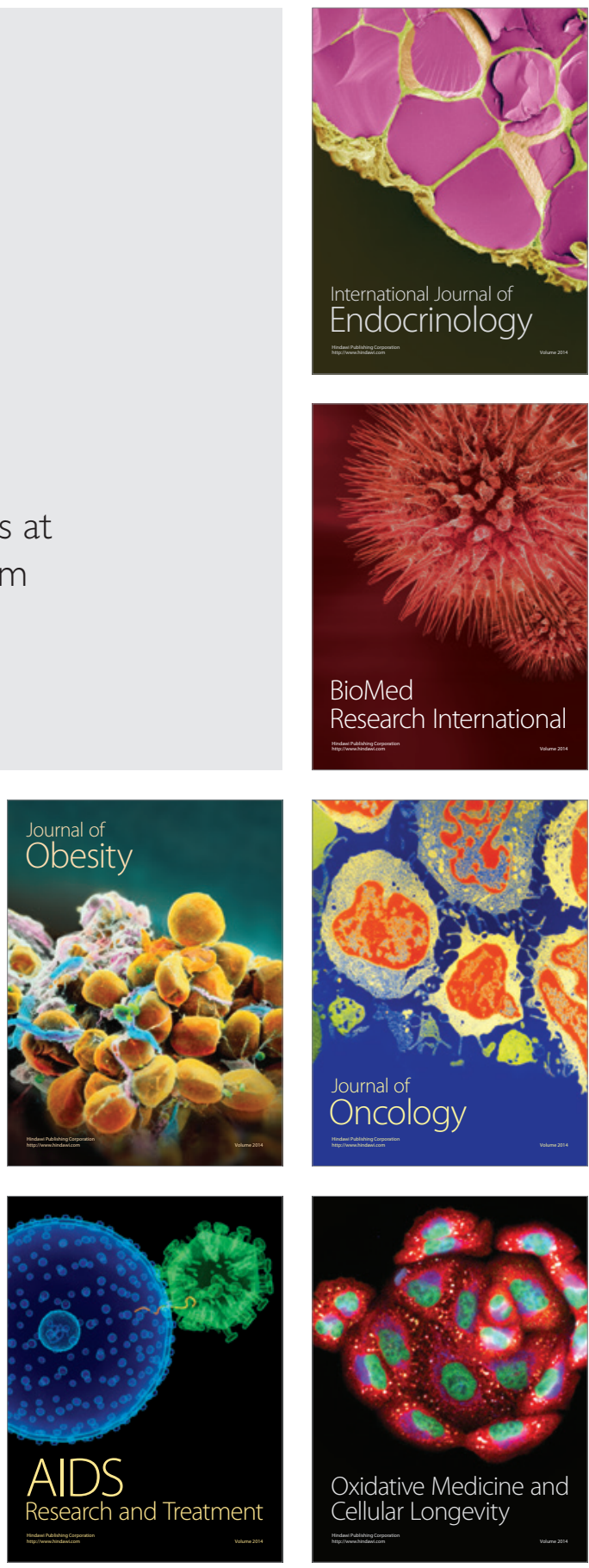